\title{
Rapid Instant Thin-Layer Chromatography System for Determining the Radiochemical Purity of ${ }^{68} \mathrm{Ga}$-DOTATATE
}

\author{
Michael G. Bornholdt, Kayla M. Woelfel, Ping Fang, Mark S. Jacobson, and Joseph C. Hung \\ Division of Nuclear Medicine, Department of Radiology, Mayo Clinic, Rochester, Minnesota
}

The objective of this study was to develop instant thin-layer chromatography (ITLC) conditions for the determination of radiochemical purity of ${ }^{68} \mathrm{Ga}$-DOTATATE in a shorter time period than those stated in the NETSPOT (Advanced Accelerator Applications, Saint-Genis-Pouilly, France; AAA) kit package insert $(\mathrm{PI})$. A faster ITLC system is needed to reduce the 48- to 50 min development time so that more radioactivity is available for single patient use and wait times are shorter in the event of kit failure. Methods: Variations of the PI mobile system were evaluated with microfiber chromatography paper impregnated with silica gel (ITLC-SG). After a more suitable mobile system was identified, evaluation began by attempting to shorten the $10-\mathrm{cm}$ development distance to 7, 8, and $9 \mathrm{~cm}$. Results: Experiments using variations of PI mobile phase showed that increasing the proportion of methanol in the mobile phase decreased development time. Additionally, if the ratio of $1 \mathrm{M}$ ammonium acetate was reduced to $10 \%$ or less, retention factor values fall outside specification. Reducing the development distance shortened development time as expected; however, it also affected the resolution aspect of the radiochromatogram. Conclusion: The fastest developing ITLC system, which maintained resolution and peak shape, was methanol:1 $\mathrm{M}$ ammonium acetate $(80: 20 \mathrm{~V} / \mathrm{N})$ with ITLC-SG using a development distance of $8 \mathrm{~cm}$.

Key Words: NETSPOT; ${ }^{68} \mathrm{Ga}$-DOTATATE; instant thin layer chromatography (ITLC); radiochemical purity (RCP); positron emission tomography (PET) drug

J Nucl Med Technol 2018; 46:285-287

DOI: 10.2967/jnmt.117.200873

$\mathbf{O}$ June 1, 2016, the Food and Drug Administration approved NETSPOT (Advanced Accelerator Applications [AAA]), a kit for the preparation of ${ }^{68} \mathrm{Ga}$-DOTATATE injection. This radioactive drug is indicated for the localization of somatostatin receptor-positive neuroendocrine tumors in adult and pediatric patients (1). The package insert (PI) for NETSPOT calls for radiochemical purity (RCP) determination using glass microfiber chromatography paper impregnated with salicylic acid (ITLC-SA) (1). However, this ITLC-SA method takes approximately $50 \mathrm{~min}$ to complete. The long

Received Aug. 14, 2017; revision accepted Apr. 5, 2018.

For correspondence contact: Joseph C. Hung, Department of Radiology, Mayo Clinic, 200 First St. SW, Rochester, MN 55905-0001.

E-mail: jhung@mayo.edu

Published online May 3, 2018

COPYRIGHT @ 2018 by the Society of Nuclear Medicine and Molecular Imaging. development time lengthens the overall drug preparation time and therefore PET imaging operations.

A PET imaging area is a fast-paced working environment that often has a regimented schedule. A disrupted schedule leads to longer waiting times for patients, who may themselves have tight clinic schedules. A significant amount of radioactivity is also lost via radioactive decay using the ITLC method in the PI. When a ${ }^{68} \mathrm{Ga}$ generator is getting weaker, this loss of radioactivity becomes an increasingly visible issue.

A more rapid ITLC method is needed to determine the RCP of ${ }^{68} \mathrm{Ga}$-DOTATATE. A shorter RCP time would maximize the amount of ${ }^{68} \mathrm{Ga}$-DOTATATE available for patient use and shorten waiting times in the event of, for example, a NETSPOT kit failure. Therefore, the objective of this study was to develop ITLC conditions for determination of the RCP of ${ }^{68} \mathrm{Ga}$-DOTATATE in a shorter time period than that stated in the PI (I).

\section{MATERIALS AND METHODS}

\section{Radiolabeling of ${ }^{68} \mathrm{Ga}$-DOTATATE}

NETSPOT comes with a reaction vial containing lyophilized DOTATATE $(40 \mu \mathrm{g}) ; 5 \mu \mathrm{g}$ of 1,10 phenanthroline; $6 \mu \mathrm{g}$ of gentisic acid; $20 \mathrm{mg}$ mannitol; and a buffer vial of reaction buffer solution $(\sim 1 \mathrm{~mL})$ containing $60 \mathrm{mg}$ of formic acid, $56.5 \mathrm{mg}$ of sodium hydroxide, and water for injection $(1,2)$. A $0.2-\mu \mathrm{m}$ vent filter assembly and a needle, attached to a $0.22-\mu \mathrm{m}$ vented filter and ${ }^{68} \mathrm{Ge}$ trapping cartridge (660 $\mathrm{mg}$ of porous silica), connected to a ${ }^{68} \mathrm{Ge} /{ }^{68} \mathrm{Ga}$ generator (Galliapharm; Eckert \& Ziegler Radiopharma $\mathrm{GmbH}$ ) are inserted into the reaction vial septum within a lead pig. DOTATATE is radiolabeled by eluting the ${ }^{68} \mathrm{Ge} /{ }^{68} \mathrm{Ga}$ generator with $5 \mathrm{~mL}$ of $0.1 \mathrm{M}$ sterile ultra-pure $\mathrm{HCl}$ eluant (Eckert \& Ziegler Radiopharma GmbH) $(1,2)$.

The PI dictates that processing take place in an aseptic environment; therefore, all production hardware and all processing take place within an ISO $5 \mathrm{~A}^{2}$ PET Dispensing Isolator (AMERCARE LTD; Thame) $(1,2)$. Immediately after elution, $0.5 \mathrm{~mL}$ of the NESPOT kit buffer is added to the reaction vial, followed by heating $(1,2)$. The heating portion of the drug labeling is completed using heater block for $8 \mathrm{~min}$ at $98^{\circ} \mathrm{C}$, the temperature set point $(1,2)$. The heater wells are protected from the flows inside the hood using an in-house fabricated Plexiglas cover. After heating, the reaction vial is cooled for $10 \mathrm{~min}$ per the PI $(1,2)$. After cooling, a small sample is withdrawn from the reaction vial for quality control (QC), which includes $\mathrm{pH}$ testing, visual inspection, radionuclidic identity, membrane filter integrity, bacterial endotoxins test, retrospective sterility testing, and RCP testing $(1,2)$. 


\section{RCP Testing Methods}

The NETSPOT PI calls for methanol:1 $\mathrm{M}$ ammonium acetate $(50: 50 \mathrm{~V} / \mathrm{V})$ as the mobile phase using ITLC-SA chromatography paper (1). In January 2017, AAA amended the PI to include glass microfiber chromatography paper impregnated with silica gel (ITLC-SG) using the same mobile phase conditions (2). ITLC-SG offers a faster development time (3). A sample was spotted $1 \mathrm{~cm}$ from the bottom of the strip using a capillary syringe and developed a distance of $10 \mathrm{~cm}$ from the point of application, within glass development tanks and 3-4 mm of mobile phase.

After ITLC development, RCP and retention factor $\left(\mathrm{R}_{\mathrm{f}}\right)$ values were determined by scanning strips with a radiometric ITLC scanner (AR2000; Eckert \& Ziegler Radiopharma $\mathrm{GmbH}$ ). $\mathrm{R}_{\mathrm{f}}$ is defined as the ratio of the distance traveled (determined by measurement of a radio-peak centroid) to the distance traveled by the solvent (solvent front). $R_{f}$ values and RCPs were calculated by identification and integration of chromatogram peaks. RCP acceptance criteria in the PI are ${ }^{68} \mathrm{Ga}$-DOTATATE $\geq 92 \%$ and other Ga species $\leq 5 \%$ $(1,2)$. Chromatograms were generated for each ITLC system tested. Two chromatogram examples of runs using methods in the updated PI (2) and the final optimal conditions are illustrated in Figure 1.

\section{Preparations of Tested Samples}

Samples used to spot strips are categorized into 2 groups: ${ }^{68} \mathrm{GaCl}_{3}$ (generator eluate; free ${ }^{68} \mathrm{Ga}$ ) and $\geq 95 \% \mathrm{RCP}{ }^{68} \mathrm{Ga}$-DOTATATE.

\section{Acceptable RCP Value}

An RCP of 95\% was selected as the minimum acceptance limit because to pass the NETSPOT RCP specification, the total ${ }^{68} \mathrm{Ga}$ species (other than the intended labeled product), must be in a total concentrations of $\leq 5 \%$. In actuality, the NETSPOT PI states that $\geq 92 \%{ }^{68} \mathrm{Ga}$-DOTATATE is needed to pass the RCP specification $(1,2)$. In the case of a sample with an RCP of $92 \%$, a single radio-

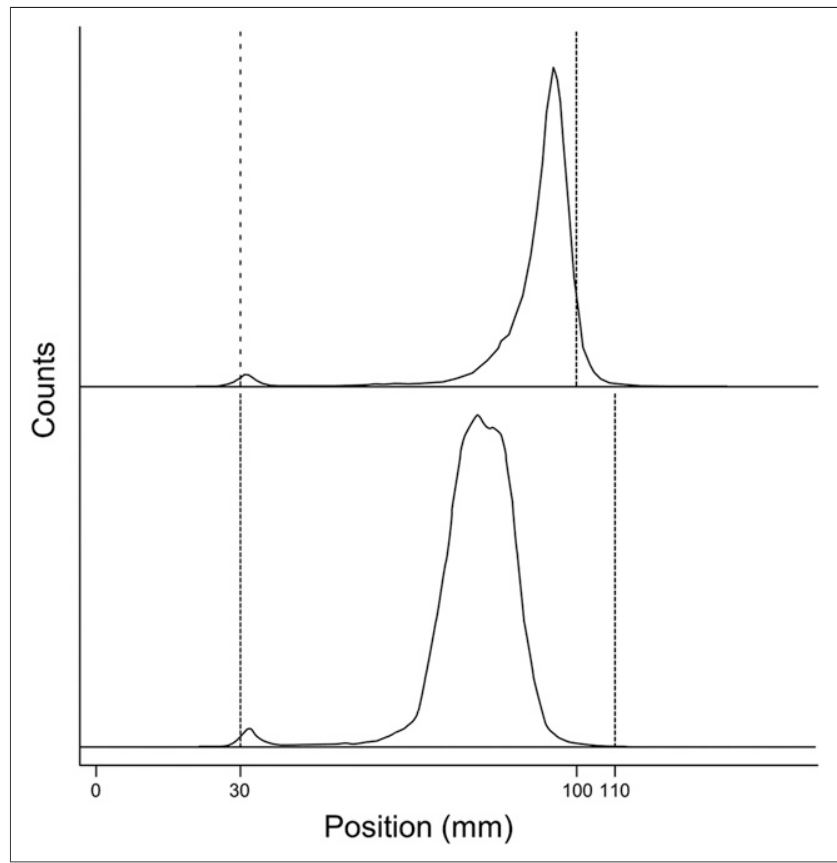

FIGURE 1. Radiochromatograms of methanol:1 $\mathrm{M}$ ammonium acetate $(50: 50 \mathrm{v} / \mathrm{v}) /$ ITLC-SG system with a development distance of $70 \mathrm{~mm}$ (top) and methanol:1 M ammonium acetate $(80: 20 \mathrm{v} / \mathrm{v}) /$ ITLC-SG system with a development distance of $80 \mathrm{~mm}$ (bottom). Dotted lines represent origin and solvent front, respectively. chemical impurity making up $8 \%$ of the sample would violate the acceptance criteria that a single ${ }^{68} \mathrm{Ga}$ species must be $\leq 5 \%(1,2)$.

\section{Tested Stationary Phase}

ITLC-SG was used in our study due to its faster development time (3).

\section{Tested Mobile Phases}

Various mobile phases were tested, which are described in Table 1.

\section{Tested Conditions for ITLC Methods}

Two criteria need to be met by an ITLC system to fulfill the objective of this study. The system needs to develop more rapidly relative to the conditions used for the PI and revised PI. Also ITLC system radiochromatography must have sufficient resolution $\left(\mathrm{R}_{\mathrm{s}}\right)$ between radioactive species.

All tested conditions are depicted in Tables 1 and 2. ITLC-SG was investigated in combination with a wider selection of mobile phases once it was determined that ITLC-SG development time was generally faster.

$\mathrm{R}_{\mathrm{S}}$ is included in Table 1 as a metric for ITLC system performance. $R_{s}$ is calculated using the following equation (4):

$$
\mathrm{Rs}=\frac{(\mathrm{Zs} 2-\mathrm{Zs} 1)}{(0.5(\mathrm{~W} 1+\mathrm{W} 2))}
$$

Zs2 and Zs1 are the migration distances of the 2 components, and $\mathrm{W} 1$ and $\mathrm{W} 2$ are the corresponding widths at the bases of the peaks. $R_{s}=1.25$ is sufficient for quantitative measurements using radio-thin-layer chromatography. In the case of fronting or tailing peaks, $R_{s} \geq 2.5$ is required for reliable quantification.

\section{Alternative Development Distances}

It was anticipated that the developing time would be reduced if the development distance was shortened. Various development distances were tested (Table 2). Nevertheless, we were in-terested in finding out whether the ITLC-SG with a shorter development distance also provided a suitable $R_{s}$ on top of time reduction.

\section{RESULTS}

\section{Mobile Phases}

Results of various mobile phases are shown in Table 1. The ITLC-SG system with methanol:1 $\mathrm{M}$ ammonium acetate

TABLE 1

RCP Evaluation Outcomes with Various Mobile Phases

\begin{tabular}{ccccc}
\hline & \multicolumn{4}{c}{ ITLC-SG paper } \\
\cline { 2 - 5 } $\begin{array}{c}\text { Mobile } \\
\text { phase }\end{array}$ & $\begin{array}{c}\text { Impurity } \\
\mathrm{R}_{\mathrm{f}}\end{array}$ & $\begin{array}{c}{ }^{68 \mathrm{Ga}-} \\
\text { DOTATATE } \\
\mathrm{R}_{\mathrm{f}}\end{array}$ & $\begin{array}{c}\text { Development } \\
\text { time }(\mathrm{min}) \\
(n=3)\end{array}$ & $\mathrm{R}_{\mathrm{s}}$ \\
\hline $\begin{array}{c}\text { Methanol:1 } \mathrm{M} \\
\text { ammonium }\end{array}$ & & & \\
acetate & & & & \\
$50: 50$ & $0.0-0.1$ & $0.8-1.0$ & $25.3 \pm 1.7$ & 2.3 \\
$60: 40$ & $0.0-0.1$ & $0.8-1.0$ & $23.9 \pm 0.5$ & 2.2 \\
$80: 20$ & $0.0-0.1$ & $0.6-0.9$ & $18.6 \pm 0.4$ & 2.1 \\
$90: 10$ & $0.0-0.1$ & $0.3-0.6$ & $15.1 \pm 0.3$ & 0.6 \\
$95: 5$ & $0.0-0.1$ & $0.1-0.3$ & $13.9 \pm 0.3$ & $\mathrm{NA}$ \\
$100: 0$ & $0.0-0.1$ & $0.0-0.1$ & $10.3 \pm 0.2$ & $\mathrm{NA}$ \\
$0: 100$ & $0.0-0.1$ & $0.0-0.1$ & $10.3 \pm 0.6$ & $\mathrm{NA}$ \\
& & & &
\end{tabular}

$N A=$ unable to identify 2 peaks on ITLC-SG. 
TABLE 2

Comparison of Standard and Alternative RCP Testing Methods

\begin{tabular}{cccc}
\hline & \multicolumn{3}{c}{ ITLC-SG $(n=3)$} \\
\cline { 2 - 4 } Mobile phase & $\begin{array}{l}\text { Development } \\
\text { distance }(\mathrm{cm})\end{array}$ & $\begin{array}{c}\text { Time } \\
(\mathrm{min})\end{array}$ & $\mathrm{R}_{\mathrm{s}}$ \\
\hline Methanol:1 M & & & \\
ammonium acetate & & & \\
50:50 & 10 & $25.3 \pm 1.7$ & 2.3 \\
& 9 & $21.2 \pm 0.3$ & 1.6 \\
& 8 & $16.1 \pm 0.3$ & 1.5 \\
& 7 & $13.3 \pm 0.2$ & 1.5 \\
\hline $80: 20$ & 10 & $18.6 \pm 0.4$ & 2.1 \\
& 9 & $15.4 \pm 0.2$ & 1.5 \\
& 8 & $12.7 \pm 0.2$ & 1.4 \\
& 7 & $10.3 \pm 0.2$ & 0.8 \\
\hline
\end{tabular}

$(80: 20 \mathrm{~V} / \mathrm{V})$ scored a relatively high mark in each of the 4 evaluated elements (i.e., radiochemical impurities $\mathrm{R}_{\mathrm{f}},{ }^{68} \mathrm{Ga}-$ DOTATATE RCP, development time, and $\mathrm{R}_{\mathrm{s}}$ ). The $\mathrm{R}_{\mathrm{f}}$ value of generator eluate (i.e., free ${ }^{68} \mathrm{Ga}$ ) was predominantly located at the baseline (i.e., $\mathrm{R}_{\mathrm{f}}<0.1$ ).

\section{Alternative Development Distances}

As shown in Table 2, the PI RCP testing method with a 7-cm development distance had the shortest development time in the group of methanol:1 $\mathrm{M}$ ammonium acetate (50:50 V/V). The $\mathrm{R}_{\mathrm{s}}$ value for this system was acceptable (i.e., 1.5) (Table 2).

Although the 7-cm RCP testing system with $80: 20 \mathrm{~V} / \mathrm{V}$ methanol:1 $\mathrm{M}$ ammonium acetate was the fastest of all tested samples, its resolution power was not adequate (i.e., $\mathrm{R}_{\mathrm{s}}=0.8$ ) (Table 2).

Judging from the development time and resolution capability, the optimized conditions of this evaluation was the RCP testing method with $8-\mathrm{cm}$ development distance and mobile phase of methanol:1 $\mathrm{M}$ ammonium acetate (80:20 V/V) (Table 2).

\section{DISCUSSION}

Reducing quality control time by shortening the RCP test is important because as a ${ }^{68} \mathrm{Ga}$ generator ages, less ${ }^{68} \mathrm{Ge}$ parent is available, leading to reduced output of ${ }^{68} \mathrm{Ga}$. After 9 mo, the quantity of ${ }^{68} \mathrm{Ge}$ will be reduced by about $50 \%$ (5). Additionally, a ${ }^{68} \mathrm{Ga}$ generator requires about $7 \mathrm{~h}$ to achieve full yield after an elution according to the summary of product characteristics (5). In the event of a kit failure, maximizing available radioactivity after a second elution, before the generator achieving its full yield, becomes critical.

In the effort to reduce time of the RCP test, widely used stationary phases were selected, which were able to separate many classes of compounds (6). It was hypothesized that ${ }^{68} \mathrm{Ga}$-DOTATATE, with its amide, amine, and hydroxyl functional groups, would adsorb more tightly to the acid structure of salicylic acid than it would to the silica gel structure (6).
In their revision to the PI (2), AAA proved the new mobile phase conditions offered faster development time. Broadly, as methanol concentration increases development time decreases while sample component separation efficiency also decreases. ITLC systems without ammonium acetate or mobile phases with $1 \mathrm{M}$ ammonium acetate concentrations $\leq 10 \%$ produce undesirable chromatograms. The ammonium acetate modifier is likely increasing efficiency of ${ }^{68} \mathrm{Ga}$-DOTATATE separation from other ${ }^{68} \mathrm{Ga}$ species, resulting in more symmetric, well-defined peaks (7).

The results in Table 1 demonstrate that methanol:1 M ammonium acetate (80:20 V/V)/ITLC-SG was the quickest developing ITLC system with $\mathrm{R}_{\mathrm{s}}$ sufficient to determine RCP. The results in Table 2 show that RCP test duration may be decreased by reducing ITLC development distance. Acceptable $\mathrm{R}_{\mathrm{s}}$ can be achieved with a development distance range of $7-10 \mathrm{~cm}$ for methanol:1 $\mathrm{M}$ ammonium acetate (50:50 V/V)/ITLC-SG, and 8-10 cm for methanol:1 M ammonium acetate $(80: 20 \mathrm{~V} / \mathrm{V}) / \mathrm{ITLC}-\mathrm{SG}$ (Table 2).

A development distance of $8 \mathrm{~cm}$ will result in the fastest time for this test under these conditions. The $R_{s}$ of this system was only slightly lower (1.4 vs. 1.5 ) than those with the speediest PI RCP testing method with a 7-cm development distance (Table 2). When compared with the most current AAA ITLC-SG conditions (3), there is an approximate 14-min decrease in development time (Table 2).

\section{CONCLUSION}

The optimal ITLC conditions for the determination of RCP was methanol:1 $\mathrm{M}$ ammonium acetate $(80: 20 \mathrm{~V} / \mathrm{V}) /$ ITLC-SG using a development distance of $8 \mathrm{~cm}$. These conditions resulted in a development time of $12.7 \pm 0.2 \mathrm{~min}$.

\section{DISCLOSURE}

Financial support for this study was provided by the Mayo Clinic PET Radiochemistry Facility, Rochester, Minnesota. No other potential conflict of interest relevant to this article was reported.

\section{REFERENCES}

1. NETSPOT. Kit for the preparation of gallium Ga 68 DOTATATE injection [package insert]. Saint-Genis-Pouilly, France: Advanced Accelerator Applications USA, Inc.; June 2016.

2. NETSPOT. Kit for the preparation of gallium Ga 68 DOTATATE injection [package insert]. Saint-Genis-Pouilly, France: Advanced Accelerator Applications USA, Inc.; January 2017.

3. Supplement approval for NETSPOT, kit for the preparation of gallium Ga 68 DOTATATE injection [letter]. U.S. Food and Drug Administration website. https://www.accessdata.fda.gov/drugsatfda_docs/appletter/2017/208547Orig1s004ltr. pdf. January 2017. Accessed July 12, 2018.

4. Spangenberg B, Poole CF, Weins C. Quantitative Thin-Layer Chromatography: A Practical Survey. Berlin, Germany: Springer-Verlag; 2011.

5. GalliaPharm Radionuclide Generator [Summary of Product Characteristics]. Berlin, Germany: Eckert \& Ziegler Radiopharma GmbH; May 2014.

6. Santiago M, Strobel S. Thin Layer Chromatography. Methods Enzymol. 2013;533:303-324.

7. Cazenave-Gassiot A, Boughtflower R, Caldwell J, et al. Effect of Increasing Concentration of ammonium acetate as an additive in supercritical fluid chromatography using $\mathrm{CO}_{2}$ : methanol mobile phase. J Chromatogr A. 2009;1216:6441-6450. 